\title{
Sex differences in emphysema phenotype in smokers without airflow obstruction
}

\author{
N. Sverzellati*, E. Calabrò̀ ${ }^{\#}$, G. Randi ${ }^{\tau,+}$, C. La Vecchia ${ }^{\top,+}$, A. Marchianò ${ }^{\S}$, \\ J-M. Kuhnigk ${ }^{f}$, M. Zompatori*, P. Spagnolo** and U. Pastorino ${ }^{\#}$
}

ABSTRACT: Data on sex differences in emphysema are limited to chronic obstructive pulmonary disease. We aimed to verify whether such differences also exist in smokers without airflow obstruction, weighting their influence on the relationship between emphysema and clinical features.

We evaluated both clinical and multidetector computed tomography (MDCT) data of 1,011 heavy smokers recruited by a lung cancer screening project. MDCT scans were analysed with software allowing lobar quantification of emphysema features. For these measures, multiple regression models were applied to assess the effect of patients sex, after allowance for age, body mass index (BMI), smoking history, forced expiratory volume in $1 \mathrm{~s}$ (FEV1) and forced vital capacity.

The final study cohort consisted of 957 smokers without airflow obstruction. Compared with males, females exhibited an emphysema phenotype less extensive in each pulmonary lobe, characterised by smaller emphysematous areas and less concentrated in the core of the lung. However, in females, the increase of emphysema with age was more pronounced and displayed a more significant relationship with $\mathrm{FEV}_{1} \%$ decline; conversely, in males there was a stronger association with the decrease in BMI.

Males and females respond differently to the type and location of lung damage due to tobacco exposure. In smokers, sex influences the relationship between emphysema and clinical features.

KEYWORDS: Cigarette smoking, computed tomography, emphysema, sex

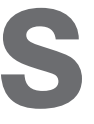
everal studies have shown the existence of sex differences in clinical presentation and phenotypes of chronic obstructive pulmonary disease (COPD) [1-4]. Furthermore, males and females seem to respond differently in type and localisation of lung damage due to tobacco exposure, suggesting that the nature of biological injury may differ between sexes [5]. In COPD, female smokers are more likely than males to exhibit small airway disease, whereas males are prone to develop a more severe emphysematous phenotype [6-8]. However, emphysema is highly variable in smokers and is not necessarily associated with COPD [9, 10]. Previous epidemiological studies have been mostly limited to COPD, giving a limited view as to the epidemiology of emphysema. Radiological investigations targeting sex-related differences are in their infancy and further investigations are warranted.

The introduction of multidetector computed tomography (MDCT) scanners has brought a new dimension to studies of emphysema, differentiating populations (i.e. by ethnicity and $\alpha_{1}$-antitrypsin deficiency) based on emphysema pattern and severity [11-14]. Pursuing a more precise phenotypic-sex definition of the emphysema component through MDCT has the potential to improve our understanding of the pathogenesis and underlying biological mechanisms of emphysema. Further attention on the sexrelated emphysema phenotype may also provide more insight into different susceptibility to tobacco smoke and relative pathophysiological consequences.

Based on previous findings of COPD [8, 15], we sought to corroborate the existence of sex differences in emphysema features even among smokers without airflow obstruction. In this retrospective analysis on a lung cancer screening population, we have explored how sex influences the emphysema phenotype by using an advanced tool for emphysema measurement in a large, prospectively studied cohort of smokers without clinical evidence of airflow obstruction. The goals of the present study were to determine: 1) whether sex differences in emphysema phenotype are different from those observed in
AFFILIATIONS

*Dept of Clinical Sciences, Division of Radiology, University of Parma, Parma,

Divisions of ${ }^{\#}$ Thoracic Surgery and

${ }^{\S}$ Radiology

National Cancer Institute

"Mario Negri Institute,

+Institute of Medical Statistics and

Biometry "G. A. Maccacaro",

University of Milano, Milan,

**Research Center on Rare Lung

Diseases (MaRP)

Dept of Respiratory Diseases, University of Modena and Reggio

Emilia, Reggio Emilia, Italy.

${ }^{f}$ Fraunhofer MEVIS-Institute for

Medical Image Computing, Bremen, Germany.

CORRESPONDENCE

N. Sverzellati

Dipartimento di Scienze Cliniche,

Sezione di Radiologia

University di Parma

Ospedale Maggiore di Parma

Padiglione Barbieri

V.Gramsci 14

43100 Parma (PR)

Italy

Fax: 390521986352

E-mail: nicolasve@tiscali.it

Received:

July 192008

Accepted after revision:

December 222008

SUPPORT STATEMENT

The present study was supported by grants from the Italian Association for Research on Cancer (AIRC) and

Lombardia-Cariplo Foundation (both Milan, Italy).

STATEMENT OF INTEREST

A statement of interest for J-M. Kuhnigk can be found at

www.erj.ersjournals.com/misc/ statements.dtl

European Respiratory Journal

Print ISSN 0903-1936

Online ISSN 1399-3003 
COPD patients; and 2) whether sex influences the relationship between emphysema and other variables, such as age, smoking history and functional decline.

\section{METHODS}

\section{Study population}

The study group consisted of 1,011 smokers (recruited consecutively by the Multicentric Italian Lung Detection (MILD, Milan , Italy) project at the National Cancer Institute of Milan between September 2005 and December 2007), whose baseline low-dose MDCT scans were available for emphysema assessment. The MILD project is an ongoing population-based randomised, controlled, lung cancer screening trial, and its primary aim is the assessment of smoking cessation rate among participants, and the impact of early lung cancer detection on mortality [16]. Eligibility criteria include age $\geqslant 49$ yrs, current or former smokers (having quit smoking within 10 yrs before recruitment) with $\geqslant 20$ pack-yrs of smoking history and no history of cancer within the previous 5 yrs. The control group was evaluated by only clinical and functional tests, whereas noncontrol cases underwent repeat low-dose MDCT scans. All subjects underwent spirometry at enrolment and a baseline standardised questionnaire to collect sociodemographic information and smoking history data. The trial was approved by the institutional review board and by the ethics committees of the centres taking part in the project. All subjects gave written informed consent.

All demographic and pulmonary function data, smoking history and emphysema assessment were recorded. Subjects were classified into modified Global Initiative for Chronic Obstructive Lung Disease staging [17]. Since spirometric data were only collected pre-bronchodilator, subjects with a forced expiratory volume in $1 \mathrm{~s}$ (FEV1)/forced vital capacity (FVC) ratio $<0.7$ were excluded from the study.

\section{Low-dose MDCT scanning}

Scanning was performed using a 16-detector row CT (Somatom Sensation 16; Siemens Medical Solutions, Forchheim, Germany). All MDCT scans of the whole lung were acquired during one deep inspiratory breath-hold without the use of contrast medium. The scanner was regularly calibrated to allow reliable measurements and comparison between examinations. Standard low-dose MDCT parameters were as follows: $120 \mathrm{kV}, 30 \mathrm{mAs}, 0.75 \mathrm{~mm}$ collimation, gantry rotation time $0.5 \mathrm{~s}$ and pitch 1.5. As for emphysema assessment, we used images reconstructed for the detection of pulmonary nodules: $1-\mathrm{mm}$ thick sections with a reconstruction increment of $1 \mathrm{~mm}$ and a sharp kernel (Siemens B50 kernel; Siemens Medical Solutions).

\section{Emphysema assessment}

All MDCT scans were transferred to a separate PC workstation and analysed for emphysema assessment by a 7-yr-experienced thoracic radiologist (N. Sverzellati). For this purpose, prototypical software (MeVis Research, Bremen, Germany) was used [18]. Briefly, this software allowed the application of high-precision 3-dimensional (3D) image analysis tools to volumetric MDCT data providing, for both the whole lung and each pulmonary lobe, a convenient assessment of MDCT parameters, such as pulmonary volumes, mean lung density (MLD), 15th percentile point (defined as the cut off value in
HU below which $15 \%$ of all voxels are distributed), emphysema proportion (using an upper threshold of $-950 \mathrm{HU}$ ) and distributional features of emphysema (i.e. core or peel predominance in the whole lung, with the peel region defined as the peripheral $10 \mathrm{~mm}$ of lung area and the remaining area defined as the core region). The emphysematous regions were further categorised according to their volumes. The 3D emphysematous volumes were sorted as follows: modified from BLECHSCHMIDT et al. [19] 2 to $<8 \mathrm{~mm}^{3}$ (class 1); 8 to $<65 \mathrm{~mm}^{3}$ (class 2); 65 to $<120 \mathrm{~mm}^{3}$ (class 3); and $>120 \mathrm{~mm}^{3}$ (class 4) [20]. The lower limit of $2 \mathrm{~mm}^{3}$ in the smallest cluster was used to minimise the influence of noise on the evaluation [21]. A $3 \times 3$ kernel-based axial Gaussian smoothing was applied to minimise the noise in sharp kernel images.

\section{Spirometry}

Lung function was measured at baseline in all study participants using a flow spirometer (KoKo; nSpire Health, Inc., Longmont, CO, USA) according to American Thoracic Society recommendations [22]. FEV1 and FVC were expressed as \% predicted value according to the European Community Lung Health Survey [23].

\section{Statistical analysis}

Sex comparisons were made using a paired t-test or Chisquared testing as appropriate. Multivariate linear regression models were used to assess the relationships between sex and the dependent variables of MDCT emphysema features. The models included potential confounding effects of age, body mass index (BMI), FEV1 \% pred, FVC \% pred, smoking status and number of pack-yrs of smoking. The significance of the interactions for the combined effect of sex with the other covariates was assessed by comparing the differences between the deviances of the models with and without the interaction term to the Chi-squared distribution with one degree of freedom. The heterogeneity test was applied to evaluate the difference between two or more estimates for the effect of patients sex on emphysema distribution and clusters. SAS Release 8.2 (SAS Institute Inc. Cary, NC, USA) was used for statistical calculation.

\section{RESULTS}

\section{Baseline demographic and clinical characteristics}

The final study cohort consisted of 957 subjects (aged $58.1 \pm 5.9$ yrs; 614 males). 54 subjects were excluded from the original study group because of a $\mathrm{FEV} 1 / \mathrm{FVC}$ ratio $<0.7$. Demographic and pulmonary function data, smoking history and MDCT emphysema features are tabulated against sex in table 1. All subjects were white Caucasians. 10 (1\%) subjects had lung cancer detected by low-dose MDCT. Females were slightly younger than males, with a slightly lower BMI, and reported a milder smoking history (table 1). Females, compared with males, exhibited similar FEV1 \% pred $(p=0.22)$, higher FVC \% pred levels ( $p=<0.0001$ ), but lower levels of forced expiratory flow at 25-75\% of FVC (FEF25-75\%) \% pred $(p=<0.0001)$ and FEV1/FVC ratio $(p=0.03)$. These associations were not substantially modified after adjusting for age, packyrs and whole-lung emphysema proportion; thus, males had significantly lower levels of FVC \% pred (-7.45\%; 95\% confidence interval (CI) $-9.66--5.24 ; \mathrm{p}<0.0001)$ and higher 


\begin{tabular}{|c|c|c|c|}
\hline Parameter & Female $^{\#}$ & Male & p-value \\
\hline \multicolumn{4}{|l|}{ Demographics } \\
\hline Age yrs & $57.56 \pm 5.9$ & $58.49 \pm 5.9$ & 0.02 \\
\hline $\mathrm{BMl} \mathrm{kg} \cdot \mathrm{m}^{-2}$ & $24.45 \pm 4.3$ & $26.64 \pm 3.7$ & $<0.0001$ \\
\hline Duration of smoking yrs & $34.92 \pm 7.3$ & $37.31 \pm 7.3$ & $<0.0001$ \\
\hline Number of cigarettes per day & $21.97 \pm 8.7$ & $24.86 \pm 10.9$ & $<0.0001$ \\
\hline Smoking pack-yrs & $38.35 \pm 17.8$ & $46.30 \pm 22.1$ & $<0.0001$ \\
\hline Time since cessation yrs & $0.93 \pm 3.14$ & $1.57 \pm 3.05$ & 0.002 \\
\hline Smoking status & & & 0.0007 \\
\hline Current smokers & $266 \pm 78.0$ & $414 \pm 67.7$ & \\
\hline FVC & $3.12 \pm 0.6$ & $4.29 \pm 0.8$ & $<0.0001$ \\
\hline FVC \% pred & $111.29 \pm 18.0$ & $103.31 \pm 16.1$ & $<0.0001$ \\
\hline FEV1/FVC ratio & $97.25 \pm 11.1$ & $98.91 \pm 12.2$ & 0.03 \\
\hline FEF25-75\% \% pred & $67.62 \pm 27.2$ & $78.33 \pm 31.2$ & $<0.0001$ \\
\hline \multicolumn{4}{|l|}{ MDCT indices } \\
\hline Lung volume $\mathrm{mL}$ & $4890.91 \pm 755.8$ & $6461.84 \pm 1.055 .8$ & $<0.0001$ \\
\hline MLD HU & $-829.42 \pm 27.6$ & $-840.05 \pm 25.5$ & $<0.0001$ \\
\hline 15th percentile point $\mathrm{HU}$ & $-914.85 \pm 21.1$ & $-923.72 \pm 17.6$ & $<0.0001$ \\
\hline \multicolumn{4}{|l|}{ Emphysema proportion \% } \\
\hline Whole lung & $3.71 \pm 4.1$ & $4.90 \pm 4.0$ & $<0.0001$ \\
\hline Whole lung core & $3.58 \pm 4.6$ & $4.91 \pm 4.4$ & $<0.0001$ \\
\hline \multicolumn{4}{|c|}{ Emphysema proportion by cluster classes \% } \\
\hline 1 & $0.63 \pm 0.5$ & $0.80 \pm 0.6$ & $<0.0001$ \\
\hline 2 & $0.65 \pm 0.6$ & $0.94 \pm 0.7$ & $<0.0001$ \\
\hline 3 & $0.11 \pm 0.1$ & $0.16 \pm 0.1$ & $<0.0001$ \\
\hline 4 & $1.54 \pm 3.4$ & $2.20 \pm 3.1$ & 0.003 \\
\hline
\end{tabular}

Data are presented as mean \pm SD, unless otherwise stated. BMI: body mass index; FEV1: forced expiratory volume in $1 \mathrm{~s} ; \%$ pred: \% predicted; FVC: forced vital capacity; FEF25-75\%: forced expiratory flow at 25-75\% of FVC; MDCT: multidetector-row computed tomography; MLD: mean lung density. ${ }^{\#:} n=343 ;{ }^{~}: n=614$.

levels of FEF25-75\% \% pred $(0.90 \%$ greater; 95\% CI $0.07-1.04$; $\mathrm{p}<0.0001)$.

\section{Emphysema assessment}

All the MDCT emphysema measurements were less severe in female than in male smokers $(p<0.0001$; table 1$)$. Such differences were evident in the univariate models and in most cases were larger in multivariate models after adjustment for age, BMI, smoking status, pack-yrs of smoking, FEV1 and FVC $\%$ pred. Table 2 summarises the differences between males and females for all variables considered. Both the MLD and the 15 th percentile point were greater in females $(p<0.0001)$.
The proportion of emphysema in females was lower in the whole lung, both in the core and peel regions. However, as shown by sex differences for the core/peel ratio, the pattern of emphysema on the axial plane slightly differed between sexes, as females showed a lower proportion of emphysema in the core of the lung compared with the peel, whereas the reverse was true in males ( $\mathrm{p}<0.0001$; fig. 1$)$. We observed that the sex differences in the lobar emphysema proportion were heterogeneous: in females emphysema proportion was lower in each lobe $(\mathrm{p}<0.0001)$, but such differences were greater in the middle lobe and in the upper lobes compared with the lower lobes ( $p=0.004$; fig. 2). Table 2 also summarises multivariate 
regression analyses of the emphysematous areas sorted by size. Both females and males showed similar proportion of the smallest clusters $(\mathrm{p}>0.05)$, although males displayed a significantly greater proportion of the bigger clusters, with the greatest one being reported for the class 4 clusters (on average $1.31 \%$ higher; $95 \%$ CI $0.89-1.73$; $\mathrm{p}<0.0001$; fig. 1 ).

\section{Relationship between emphysema and clinical features}

In the multivariate models, we observed significant interactions between sex and the other covariates, namely age, BMI and FEV1 \% pred (fig. 3). Based on these interaction analyses, in both males and females the proportion of whole-lung emphysema increased with age. This increase was higher in females and females $>68$ yrs showed no difference in emphysema extent compared with males (fig. 3a). Furthermore, by examining the mean sex differences in MLD for different combined categories of age and years of smoking in order to disclose whether sex differences in emphysema were due to a lead-time bias (i.e. females had less severe emphysema simply because they were at an earlier stage of disease than males), we observed that among subjects with 31-35, 36-40 and $>40 \mathrm{yrs}$ of smoking, the differences between males and females tended to decrease progressively with age (table 3).

As FEV1\% pred levels decreased, the whole-lung emphysema proportion tended to increase more rapidly in females than in males, and females with a FEV $1 \%$ pred $<76.2 \%$ had a greater emphysema proportion than males (fig. $3 b$ ). The total emphysema proportion tended to increase with the decrease of BMI, and the increase was more rapid in males. These data suggest that females tended to have lower emphysema proportion than males, particularly in nonoverweight subjects (BMI $<40$; fig. 3c). Despite the significant interaction between sex and FVC $\%$ pred $(\mathrm{p}<0.0001)$, females seemed to maintain a lower whole-lung emphysema proportion compared with males (within the range of the FVC \% pred levels analysed). An interaction between pack-yrs and sex was also observed $(p=0.02)$ and, in males, the whole-lung emphysema negatively correlated with pack-yrs. There was no interaction between smoking status and sex. Conversely, former smokers exhibited a greater emphysema extent compared with both male and female current smokers $(p<0.0001)$, although sex differences remained substantially the same (table 2) once the former smokers were excluded from the analysis.

\section{DISCUSSION}

The present analysis demonstrates sex-specific differences in radiological features of emphysema in a large cohort of heavy smokers without airflow obstruction. We showed through lowdose MDCT that females, compared with males, exhibited an emphysema phenotype less extensive in each pulmonary lobe and characterised by smaller emphysematous areas and slightly less concentrated in the core of the lung. Our findings substantially mirror and extend those reported in patients with COPD. Thus, an analysis limited to severe emphysematous patients from the National Emphysema Trial [8], showed that emphysema in females, relative to males, was less extensive and characterised by smaller hole size, but less peripheral involvement on CT. Furthermore, DRANSFIELD et al. [15]

TABLE 2 Estimated differences between males and females for computed tomography emphysema measurements by means of univariate and multivariate regression models

Univariate analysis

Estimated difference between males and females $(95 \% \mathrm{Cl})$
Multivariate analysis

Estimated difference between males and females $(95 \% \mathrm{Cl})$

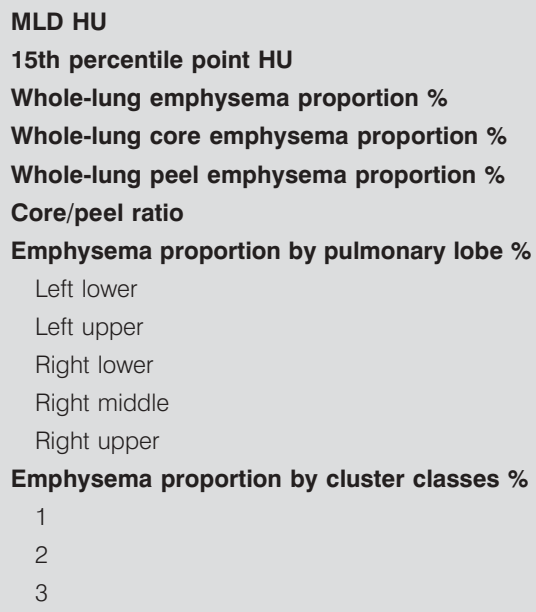

$\begin{array}{rc}-10.63(-14.11--7.16) & <0.0001 \\ -8.85(-11.35--6.35) & <0.0001 \\ 1.20(0.66-1.73) & <0.0001 \\ 1.33(0.74-1.92) & <0.0001 \\ 1.03(0.54-1.53) & <0.0001 \\ 0.12(0.08-0.17) & <0.0001 \\ & \\ 1.15(0.62-1.68) & <0.0001 \\ 1.53(0.89-2.16) & <0.0001 \\ 0.84(0.30-1.38) & 0.0023 \\ 2.19(1.41-2.98) & <0.0001 \\ 0.84(0.29-1.40) & 0.0028 \\ & <0.0001 \\ 0.166(0.092-0.240) & <0.0001 \\ 0.288(0.204-0.373) & <0.0001 \\ 0.050(0.033-0.067) & 0.0025\end{array}$

$\begin{aligned}-17.00(-20.34--13.65) & <0.0001 \\ -12.75(-15.13--10.36) & <0.0001 \\ 2.00(1.49-2.51) & <0.0001 \\ 2.10(1.54-2.66) & <0.0001 \\ 1.89(1.42-2.36) & <0.0001 \\ 0.10(0.06-0.14) & <0.0001\end{aligned}$

$1.91(1.39-2.43) \quad<0.000$

$2.48(1.86-3.09) \quad<0.0001$

$1.52(0.99-2.04) \quad<0.0001$

$3.10(2.31-3.89) \quad<0.0001$

$1.58(1.04-2.12) \quad<0.0001$

$-0.21(-0.42-0.00) \quad 0.054$

$0.40(0.31-0.48) \quad<0.0001$

$0.08(0.06-0.09) \quad<0.0001$

$1.31(0.89-1.73) \quad<0.0001$

MDCT: multidetector computed tomography; Cl: confidence interval; MLD: mean lung density. ${ }^{\#}$ : obtained from linear regression models including terms for age, body mass index, smoking status, pack-yrs of smoking, forced vital capacity and forced expiratory volume in $1 \mathrm{~s} \%$ predicted. 

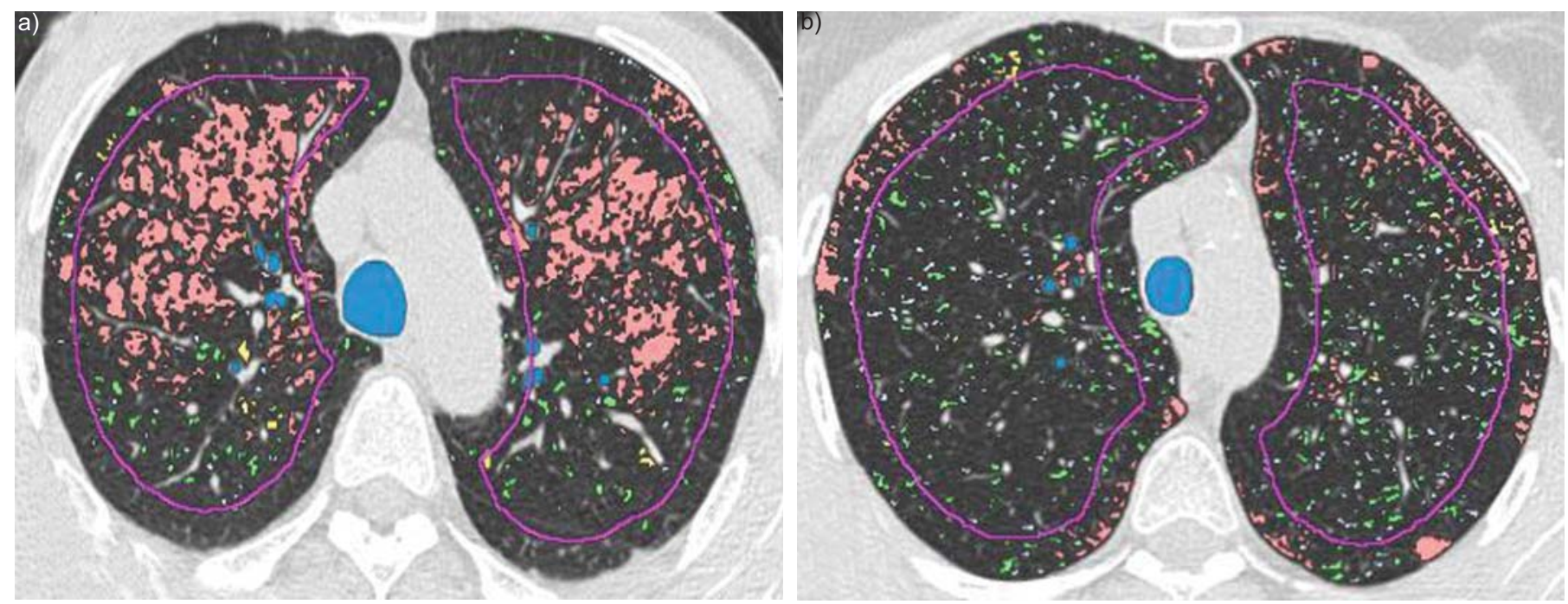

FIGURE 1. a) Colour-coded overlay of a multidetector computed tomography (CT) slice in the upper lobes of a 56-yr-old male showing the size distribution (cluster) of the emphysematous areas (largest clusters in purple, intermediate in yellow and smallest in green); the purple line delineates the core and the peel of the lung. Note that emphysema tends to spare the peel of the lung. b) CT slice of a 54-yr-old female showing a less extensive emphysema phenotype (compared with a), predominantly characterised by smaller clusters randomly distributed throughout the lung parenchyma.

demonstrated that females had less severe emphysema at all stages of COPD.

Data on the epidemiology of emphysema in "healthy" smokers are forthcoming from populations screened by lung cancer [24-26]. Both prevalence and severity of emphysema on lowdose CT have already been shown to be greater in male than in female smokers [24, 26]. We characterised emphysema using objective 3D techniques on high-resolution volumetric MDCT datasets, whereas previous studies had assessed emphysema by visual score systems, which, compared with objective methods, tend to underestimate the prevalence of low-grade emphysema [27]. Limited emphysema is usually clinically insignificant; nevertheless, we specifically looked at this subgroup to verify the hypothesis that sex differences in tobacco-smoke susceptibility exist.

Changes in the size of the emphysematous areas were reported to be useful in evaluating the pattern of progression of emphysema [28]. In the current study, it was found that the highest emphysematous clusters (class 4 ) in males represented the most striking difference among clusters between sexes, whereas males and females showed the same proportion of the smallest clusters (class 1; fig. 1). Our results are in keeping with early pathological studies demonstrating that there were no sex differences among smokers in susceptibility to microscopically assessed emphysema, whereas macroscopic emphysema was more commonly found in males $[29,30]$.

Differences in the distribution of emphysema may help understanding the disease pathogenesis and the variable development of COPD in smokers. In COPD, it has been shown that the greater proportion of emphysema in male subjects is maintained both in the upper and the lower zones of the lungs [15]. In the present cohort of smokers without airflow obstruction, males showed more severe emphysema in each lobe. However, such differences were significantly higher in the upper lobes and in the middle lobe $(\mathrm{p}<0.0001$; fig. 2$)$. This does not imply that males, compared with females, showed emphysema with a more predominant cranial distribution as the middle lobe, the left upper lobe and both the lower lobes overlap topographically with the lower and the upper pulmonary regions, respectively. Conversely, the novel finding of the middle lobe predominance may explain why smokers have a more homogeneous distribution of emphysema than previously thought $[11,31]$. To our knowledge, this is the first study that has objectively assessed emphysema in each pulmonary lobe and confirms, on a large group of subjects, that early smoking-induced emphysema tends to develop predominantly at these levels.

In females, relative to males, the emphysema was slightly less concentrated in the core of the lung (tables 1 and 2; fig. 1). This is different from what is seen in severe COPD subjects, whose emphysema was less peripheral in females, because the radial distribution of emphysema may have a functional impact, it was suggested that such difference likely accounted for the lower diffusing capacity of the lung for carbon monoxide $(D \mathrm{~L}, \mathrm{CO})$ observed in females [8]. Unfortunately, we could not explore such morphological-functional relationships as the $D \mathrm{~L}, \mathrm{CO}$ was not part of our lung screening project.

Based on the ability to adjust for the whole-lung proportion of emphysema, pack-yrs and age, we noted a similar FEV1 \% pred, but significantly lower FVC \% pred in males and lower FEF $25-75 \% \%$ pred in females. We speculated that the lower FVC \% pred in males and the lower $\mathrm{FEF} 25-75 \% \%$ pred in females were due to either the different emphysematous phenotype or by the greater degree of peripheral airways disease in females, whose smaller airway size may also place them at greater risk because toxic particulates may be more likely to deposit prior to reaching the alveoli [32]. However, such interpretation is limited by the slight differences of functional levels between sexes and by the lack of the MDCT assessment of the airway phenotype. 
a)

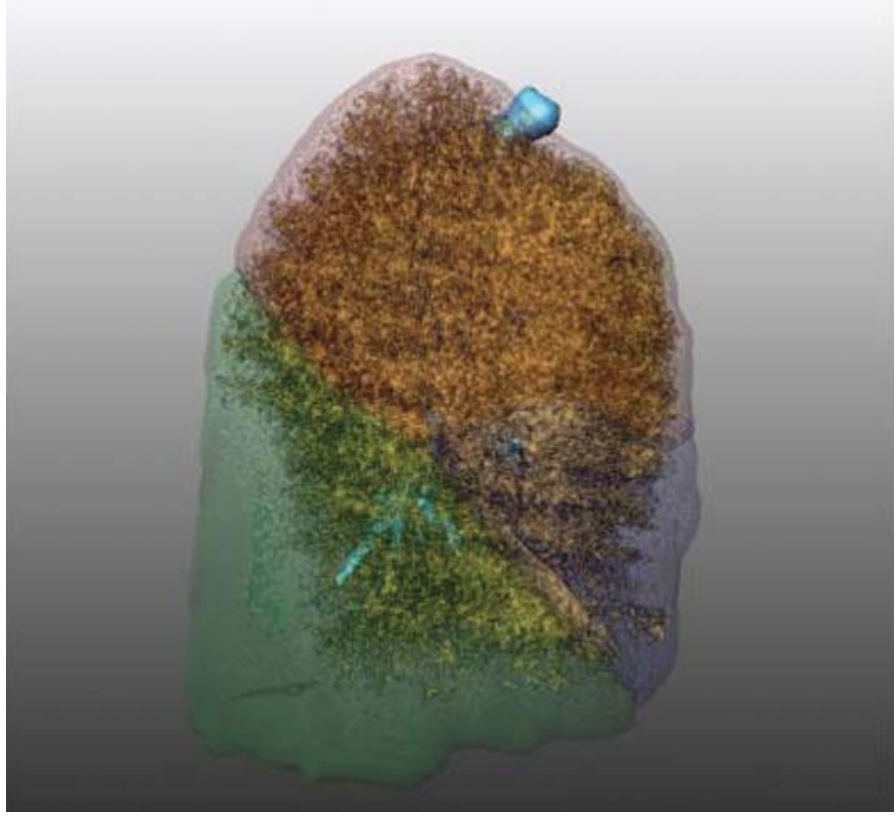

b)

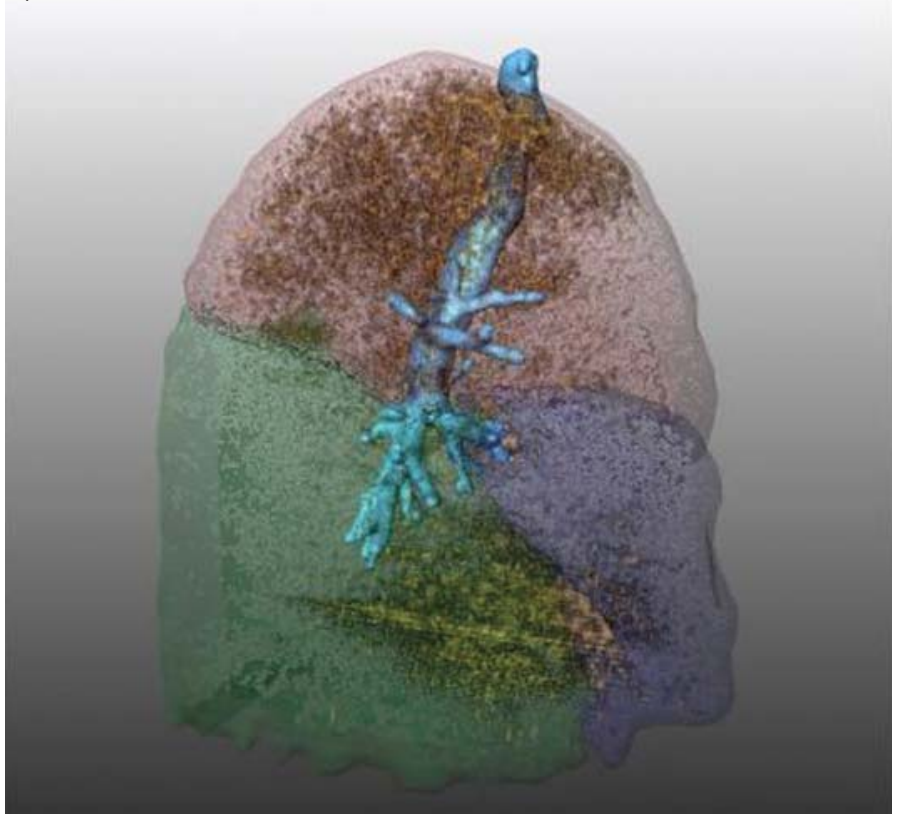

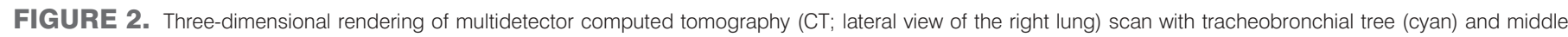

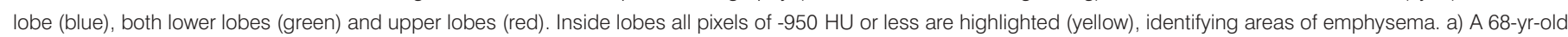
male displays a greater emphysema proportion in each lobe compared with b) a 55-yr-old female, such differences are greater in the middle and upper lobes.

These findings suggested that females tended to develop a less severe emphysematous phenotype compared with males but such sex differences were less with the increase of age, and FEV1 \% pred decline (fig. $3 a$ and b). CT densitometry may be influenced by several factors, such as age and weight [33]. However, we observed that holding constant the other covariates, as age increased, whole-lung emphysema proportion increased more rapidly in females than in males. Such a finding was also supported by the trend of sex differences in
MLD, which was shown to decrease when stratified by age and duration of smoking (table 3). Furthermore, we also observed that both the core and peel emphysema and the largest emphysematous clusters (i.e. the main sex differences of emphysema) behaved in a similar way compared with the overall emphysema extent; namely, they tended to increase more rapidly in females than in males over time (data not shown). By contrast, in males, emphysema increased more rapidly with the decrease of BMI (fig. 3c). The finding that
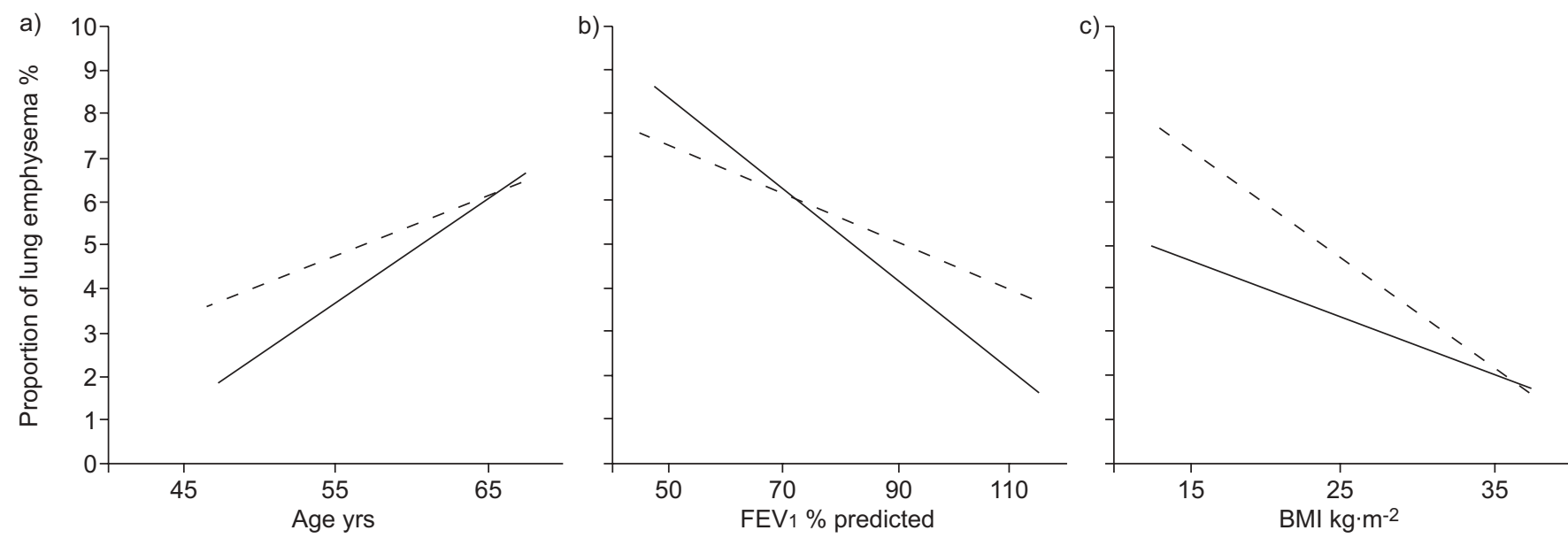

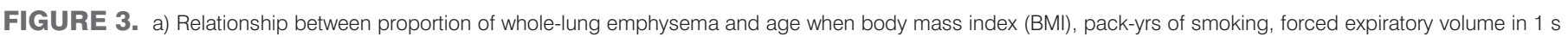

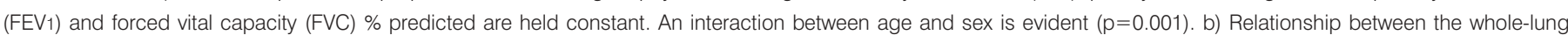

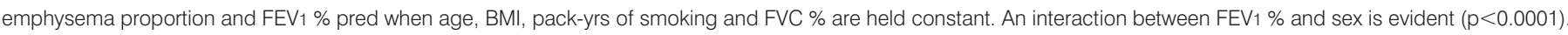

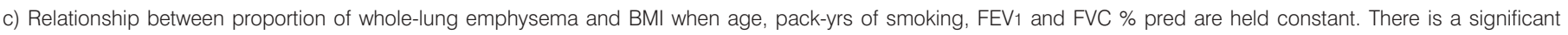
interaction between $\mathrm{BMI}$ and $\operatorname{sex}(\mathrm{p}<0.0001)$. Females: —_ males: - - - - 


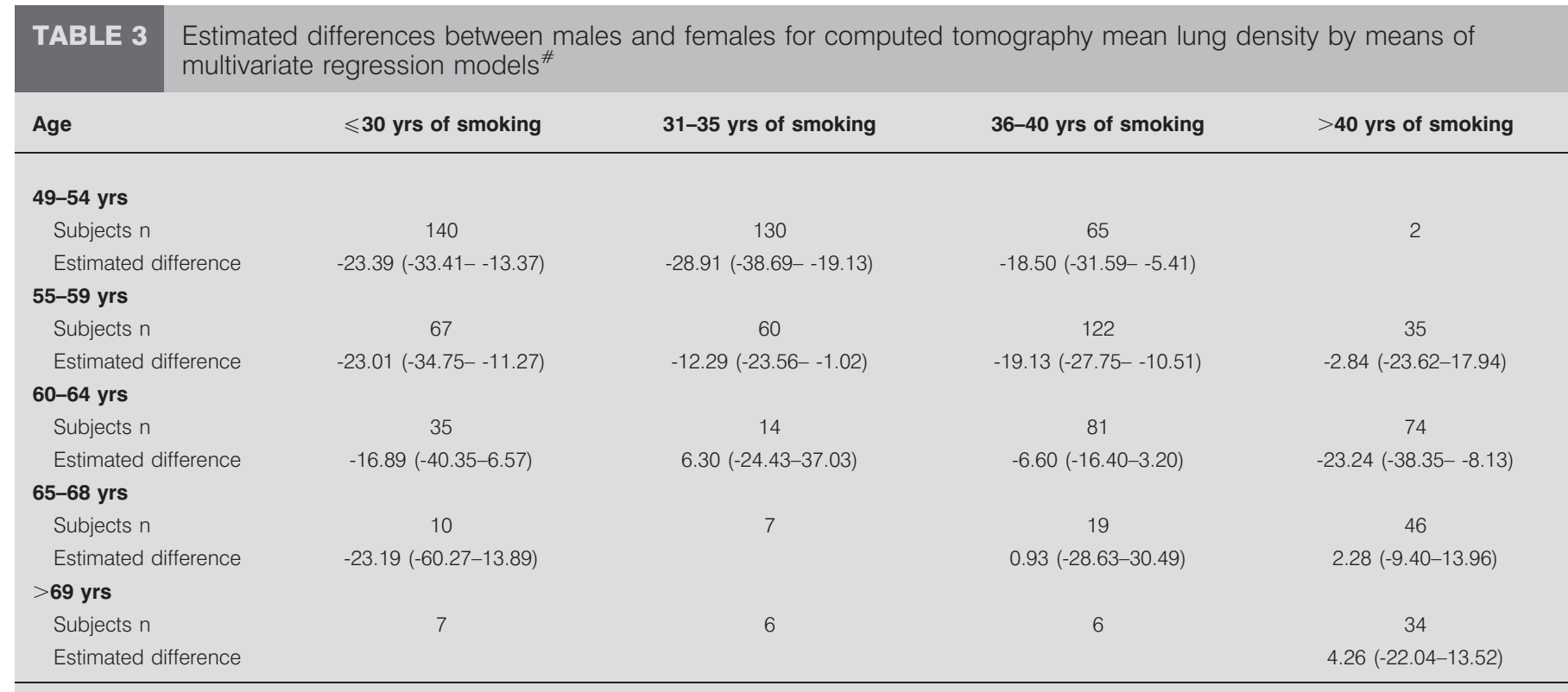

Data are presented as estimated mean sex difference in mean lung density ( $95 \%$ confidence interval), unless otherwise stated. In the smallest patient sub-groups,

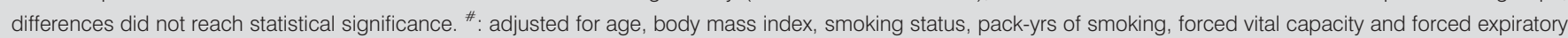
volume in $1 \mathrm{~s} \%$ predicted.

emphysema extent increases while BMI decreases, has also been reported in COPD and, importantly, may suggest a more severe systemic inflammatory response in smokers with greater emphysema extent [34]. Although no interactions were observed among severe COPD patients between sex and lung function [8], our data show that, as the FEV1 \% pred levels decreased, the emphysema extent increased more rapidly in females than in males (fig. 3b), indicating a stronger relationship between the increase in the emphysema extent and lung function deterioration in female smokers without airflow obstruction. In addition, we demonstrated that, for a given pack-yrs history, males had more severe emphysema than females. Interestingly, male smokers with higher pack-yr history displayed fewer emphysematous changes.

However, the relationship between cigarette smoking and the presence of emphysema shows a rough dose-response curve between pack-yrs of smoking and emphysema [10]. Furthermore, no interaction between smoking status and sex was observed and both female and male former smokers had more severe emphysema than current smokers. Although these observations are, at first sight, surprising, smoking cessation may be more common in those individuals with more extensive disease (i.e. healthy smoker effect). Conversely, low-grade emphysema is unlikely to lead smokers to cessation, although these findings have to be interpreted cautiously due to the patient self-reported smoking status and in the absence of any objective assessment (i.e. exhaled carbon monoxide and nicotine metabolite levels).

Our study does have significant limitations. A low-dose MDCT protocol was applied due to the fact that radiations have an intrinsic risk of inducing a neoplasm. Although a low-dose technique is now recognised as having no significant effect on the CT quantification of emphysema $[35,36]$, this may not be negligible for our population of subjects whose lungs were near-normal with limited extension of emphysema. Nevertheless, differences in both lung function test results and MLD differences would suggest that the sex differences we observed are not due to MDCT artefacts, but instead represent real findings. However, because full functional data were not available, we cannot exclude that a small minority of subjects were misclassified (individuals with restrictive defects), although there was no evidence of fibrosis on lowdose MDCT. In addition, although the analyses have been adjusted for various factors, it must be appreciated that sex differences in emphysema phenotype may be accounted for by hormonal factors, anatomical differences in airways and different smoking habits.

In conclusion, our study shows that sex differences exist in emphysema phenotype in subjects without COPD, thus suggesting a sex-specific pattern of early smoking-induced lung damage. In addition, we propose that sex analysis of emphysema according to age, BMI, pack-yrs of smoking and FEV1 \% pred may help refining emphysema phenotypes and improve our understanding of the disease pathogenesis.

\section{ACKNOWLEDGEMENTS}

The current authors are deeply grateful to C. Jacomelli (Istituto Nazionale tumouri, Milan, Italy) for their technical assistance.

\section{REFERENCES}

1 Watson L, Vestbo J, Postma DS, et al. Gender differences in the management and experience of chronic obstructive pulmonary disease. Respir Med 2004; 98: 1207-1213. 
2 de Torres JP, Campo A, Casanova C, et al. Gender and chronic obstructive pulmonary disease in high-risk smokers. Respiration 2006; 73: 306-310.

3 de Torres JP, Casanova C, Hernandez C, et al. Gender associated differences in determinants of quality of life in patients with COPD: a case series study. Health Qual Life Outcomes 2006; 4: 72.

4 Langhammer A, Johnsen R, Holmen J, et al. Cigarette smoking gives more respiratory symptoms among women than among men. The Nord-Trondelag Health Study (HUNT). I Epidemiol Community Health 2000; 54: 917-922.

5 Han MK, Postma D, Mannino DM, et al. Gender and chronic obstructive pulmonary disease: why it matters. Am J Respir Crit Care Med 2007; 176: 1179-1184.

6 Burrows B, Bloom JW, Traver GA, et al. The course and prognosis of different forms of chronic airways obstruction in a sample from the general population. $N$ Engl J Med 1987; 317: 1309-1314.

7 Tatsumi K, Kasahara Y, Kurosu K, et al. Clinical phenotypes of COPD: results of a Japanese epidemiological survey. Respirology 2004; 9: 331-336.

8 Martinez FJ, Curtis JL, Sciurba F, et al. Sex differences in severe pulmonary emphysema. Am J Respir Crit Care Med 2007; 176: 243-252.

9 Hogg JC, Wright JL, Wiggs BR, et al. Lung structure and function in cigarette smokers. Thorax 1994; 49: 473-478.

10 Hogg JC. Pathophysiology of airflow limitation in chronic obstructive pulmonary disease. Lancet 2004; 364: 709-721.

11 Stavngaard T, Shaker SB, Dirksen A. Quantitative assessment of emphysema distribution in smokers and patients with $\alpha 1$-antitrypsin deficiency. Respir Med 2006; 100: 94-100.

12 Hoffman EA, Simon BA, McLennan G. State of the art. A structural and functional assessment of the lung via multidetector-row computed tomography: phenotyping chronic obstructive pulmonary disease. Proc Am Thorac Soc 2006; 3: 519-532.

13 Dransfield MT, Davis JJ, Gerald LB, et al. Racial and gender differences in susceptibility to tobacco smoke among patients with chronic obstructive pulmonary disease. Respir Med 2006; 100: 1110-1116.

14 Shaker SB, Maltbaek N, Brand P, et al. Quantitative computed tomography and aerosol morphometry in COPD and alpha1-antitrypsin deficiency. Eur Respir J 2005; 25: 23-30.

15 Dransfield MT, Washko GR, Foreman MG, et al. Gender differences in the severity of CT emphysema in COPD. Chest 2007; 132: 464-470.

16 Bach PB, Jett JR, Pastorino U, et al. Computed tomography screening and lung cancer outcomes. JAMA 2007; 297: 953-961.

17 Rabe KF, Hurd S, Anzueto A, et al. Global strategy for the diagnosis, management, and prevention of chronic obstructive pulmonary disease: GOLD executive summary. Am J Respir Crit Care Med 2007; 176: 532-555.

18 Kuhnigk JM, Dicken V, Zidowitz S, et al. Informatics in radiology (infoRAD): new tools for computer assistance in thoracic CT. Part 1. Functional analysis of lungs, lung lobes, and bronchopulmonary segments. Radiographics 2005; 25: 525-536.

19 Blechschmidt RA, Werthschutzky R, Lorcher U. Automated CT image evaluation of the lung: a morphologybased concept. IEEE Trans Med Imaging 2001; 20: 434-442.

20 Achenbach T, Weinheimer O, Buschsieweke C, et al. Fully automatic detection and quantification of emphysema on thin section MD-CT of the chest by a new and dedicated software. Rofo 2004; 176: 1409-1415.

21 Zaporozhan J, Ley S, Eberhardt R, et al. Paired inspiratory/ expiratory volumetric thin-slice CT scan for emphysema analysis: comparison of different quantitative evaluations and pulmonary function test. Chest 2005; 128: 3212-3220.

22 Standards for the diagnosis and care of patients with chronic obstructive pulmonary disease. American Thoracic Society. Am J Respir Crit Care Med 1995; 152: S77-S121.

23 Roca J, Burgos F, Sunyer J, et al. References values for forced spirometry. Group of the European Community Respiratory Health Survey. Eur Respir J 1998; 11: 1354-1362.

24 Wang Q, Takashima S, Wang JC, et al. Prevalence of emphysema in individuals who underwent screening CT for lung cancer in Nagano prefecture of Japan. Respiration 2001; 68: 352-356.

25 Omori H, Nakashima R, Otsuka N, et al. Emphysema detected by lung cancer screening with low-dose spiral CT: prevalence, and correlation with smoking habits and pulmonary function in Japanese male subjects. Respirology 2006; 11: 205-210.

26 Hatayama O, Kobayashi T, Fujimoto K, et al. Utility of single-slice high-resolution $\mathrm{CT}$ in upper lung field combined with low-dose spiral CT for lung-cancer screening in the detection of emphysema. Intern Med 2007; 46: 1519-1525.

27 Miller RR, Muller NL, Vedal S, et al. Limitations of computed tomography in the assessment of emphysema. Am Rev Respir Dis 1989; 139: 980-983.

28 Mishima M, Hirai $\mathrm{T}$, Itoh $\mathrm{H}$, et al. Complexity of terminal airspace geometry assessed by lung computed tomography in normal subjects and patients with chronic obstructive pulmonary disease. Proc Natl Acad Sci USA 1999; 96: 8829-8834.

29 Gillooly M, Lamb D. Microscopic emphysema in relation to age and smoking habit. Thorax 1993; 48: 491-495.

30 Thurlbeck WM, Ryder RC, Sternby N. A comparative study of the severity of emphysema in necropsy populations in three different countries. Am Rev Respir Dis 1974; 109: 239-248.

31 Hersh CP, Washko GR, Jacobson FL, et al. Interobserver variability in the determination of upper lobe-predominant emphysema. Chest 2007; 131: 424-431.

32 Bennett WD, Zeman KL, Kim C. Variability of fine particle deposition in healthy adults: effect of age and gender. Am J Respir Crit Care Med 1996; 153: 1641-1647.

33 Gevenois PA, Scillia P, de Maertelaer V, et al. The effects of age, sex, lung size, and hyperinflation on CT lung densitometry. AJR Am J Roentgenol 1996; 167: 1169-1173.

34 Makita H, Nasuhara Y, Nagai K, et al. Characterisation of phenotypes based on severity of emphysema in 
chronic obstructive pulmonary disease. Thorax 2007; 62: 932-937.

35 Madani A, De Maertelaer V, Zanen J, et al. Pulmonary emphysema: radiation dose and section thickness at multidetector CT quantification-comparison with macroscopic and microscopic morphometry. Radiology 2007; 243: 250-257.

36 Gierada DS, Pilgram TK, Whiting BR, et al. Comparison of standard- and low-radiation dose CT for quantification of emphysema. AJR Am J Roentgenol 2007; 188: 42-47. 